\title{
Olsenella uli
}

National Cancer Institute

\section{Source}

National Cancer Institute. Olsenella uli. NCI Thesaurus. Code C86628.

A species of anaerobic, Gram-positive, rod shaped bacteria assigned to the phylum

Actinobacteria. This species is non-spore forming, catalase and indole negative, does not liquefy gelatin, hydrolyze hippurate, digest meat, or reduce nitrate, but does ferment glucose, glycogen, trehalose, and starch, as well as hydrolyze esculin. O. uli, also known as Lactobacillus uli, is found in human ging ival crevices and periodontal pockets where it is associated with periodontal disease. 